\title{
Density functional theory study of self-trapped holes in disordered $\mathrm{SiO}_{2}$
}

\author{
Matteo Farnesi Camellone, ${ }^{1,2}$ Thomas D. Kühne, ${ }^{3}$ and Daniele Passerone ${ }^{1}$ \\ ${ }^{1}$ Electronics/Metrology and Nanotech@Surfaces Laboratories, Swiss Federal Laboratories for Materials Testing and Research, \\ Ueberlandstrasse 129, CH-8600 Duebendorf, Switzerland \\ ${ }^{2}$ Theory@Elettra Group, INFM-CNR DEMOCRITOS, c/o Sincrotrone Trieste-SS14, Km 163, 5, Basovizza I-34012, Italy \\ ${ }^{3}$ Department of Physics and School of Engineering and Applied Sciences, Harvard University, Cambridge, Massachusetts 02138, USA
}

(Received 3 March 2009; revised manuscript received 9 June 2009; published 15 July 2009)

\begin{abstract}
The structures of self-trapped holes ( $\mathrm{STHs}$ ) in a disordered $\mathrm{SiO}_{2}$ network are investigated using firstprinciples calculations. We consider two different kinds of STHs: STH1, consisting of a hole trapped on an oxygen atom bridging two silicon atoms, and STH2, a metastable structure, in which the hole is delocalized over two bridging oxygen atoms. Using density functional theory and a self-interaction correction scheme, we fully optimize the structural model in an unbiased way and are able to reproduce, for the first time, the experimentally observed STH2 at a density functional theory level and without applying any geometrical constraint to the system. In addition, extensive molecular-dynamics simulations $(\simeq 200 \mathrm{ps})$ are performed in order to describe the hopping of a hole within the dioxide network.
\end{abstract}

DOI: 10.1103/PhysRevB.80.033203

PACS number(s): 71.55.Jv, 61.43.Dq, 72.20.Jv

The formation of defects created by radiation-induced electronic excitations changes the electronic and optical properties of silica-based materials. ${ }^{1}$ Among these defects self-trapping of holes play an important role in the radiationinduced defect formation. ${ }^{1,2}$ Therefore understanding the nature of charge traps is crucial for reliable fabrication of radiation-resistant semiconductor devices and optical fibers. An important class of intrinsic defects within insulators is the one which arises from carrier self-trapping within a topologically defect-free network. Self-trapping has been for the first time observed in alkali halides, in which the " $\mathrm{V}_{k}$ center" arises when a hole localizes on two halogen atoms through a large local lattice relaxation.,

In metal-oxide semiconductors (MOS), holes typically move hopping from site to site of the oxide until they reach the silicon-oxide interface. Here many of the holes are trapped by defects near the $\mathrm{Si}-\mathrm{SiO}_{2}$ interface and generate a fixed positive charge, which can affect the characteristics of the transistor. The description of the mechanism of hopping at a microscopic level has, therefore, great importance for a more complete understanding of possible breakdown mechanisms. In this paper both experimentally observed selftrapped holes (STHs) will be described with the help of density functional theory (DFT), and the hole hopping mechanism will be investigated with ab initio molecular dynamics (MD). STHs in silicon dioxide $\left(\mathrm{SiO}_{2}\right)$ have been predicted for the first time by $\mathrm{Mott}^{5}$ while the electron-spinresonance (ESR) experiments conducted by Griscom ${ }^{6}$ have led to the identification of two different kinds of STHs in amorphous $\mathrm{SiO}_{2}$ : one (STH1) in which the hole localizes on a single bridging oxygen and another (STH2) in which the unpaired electron is shared between two bridging oxygen atoms.

From a theoretical point of view the first studies on STH1 have been carried out by Chernov et al. ${ }^{7}$ and by Edwards. ${ }^{8}$ Edwards made use of a semiempirical Hamiltonian and unrestricted Hartree-Fock (UHF) calculations, and was able to reproduce the STH1 defect, thus supporting the model proposed by Griscom. Nevertheless, these approaches failed in describing the STH2 configuration. Approximated exchange- correlation functionals in standard DFT implementation fail to cancel exactly the self-interaction that is present in the Coulomb energy (the so-called self-interaction error). This leads to the delocalization of the hole on all the oxygen atoms of the network; on the other hand, UHF theory completely neglects correlation effects and overestimates the process of localization. A successful attempt of getting the best of these two worlds has been recently attained by Kimmel $e t$ $a l .{ }^{9}$ and by Pacchioni and co-workers. ${ }^{10,11}$ In Ref. 9 Kimmel et al. studied the STH1 using a sophisticated embedding method and a specially designed hybrid functional. From this study it seems that an accurate embedding is less important than the choice of the proper functional. Three hybrid functionals have been used in Refs. 11-13 with different amounts of exact exchange. It has been found that the hybrid functional B50-LYP (with 50\% of exact exchange) accurately reproduces the STH1 defect structure. However, the STH2 configuration obtained by these authors (keeping an angle fixed to selected values), though in agreement with EPR experiments, was not stable with respect to structural relaxation.

In the present study we use a different strategy for a complete characterization of structural and dynamic properties of the STH's defects. After a presentation of distinct UHF results in a periodic geometry, we employ DFT calculations with an empirical self-interaction correction (SIC) scheme and we are able to predict both STH1 and STH2 without applying any constraints to the system. In addition, by performing quantum MD simulations, we investigate the interesting dynamics of the mechanism by which the hole hops between two oxygen atoms.

In all our calculations periodic boundary conditions (PBC) are employed. Whereas the introductory $a b$ initio UHF calculations have been performed using the CRYSTAL06 code, ${ }^{14}$ our first-principles calculations are based on SICDFT (Ref. 15) in the implementation of VandeVondele et $a l .{ }^{16}$ The DFT calculations are performed using a mixed Gaussian and plane-waves approach ${ }^{17}$ as implemented in CP2K. ${ }^{18}$ Norm-conserving Goedecker-Teter-Hutter ${ }^{19,20}$ pseudopotentials are used and the exchange and correlation 
TABLE I. Structural properties and EPR features of the configurations obtained from SIC-DFT calculations. UHF results are included for reference.

\begin{tabular}{lcc}
\hline \hline & SIC/UHF STH1 & SIC STH2 \\
\hline Spin population $\mathrm{O}_{1}$ & $0.91\left(\mathbf{1 . 0 2}^{\mathrm{d}}\right)$ & 0.40 \\
Spin population $\mathrm{O}_{2}$ & $0.00\left(\mathbf{0 . 0 0}^{\mathrm{d}}\right)$ & 0.49 \\
$\mathrm{Si}-\mathrm{O}_{1}(\AA)\left[1.61^{\mathrm{c}}\right]$ & $1.78,1.80\left(\mathbf{1 . 8 0}^{\mathrm{d}}, \mathbf{1 . 7 8}\right)$ & $1.67^{\mathrm{a}}, 1.69$ \\
$\mathrm{Si}-\mathrm{O}_{2}(\AA)\left[1.61^{\mathrm{c}}\right]$ & $1.62,1.66\left(\mathbf{1 . 5 9}^{\mathrm{d}}, \mathbf{1 . 6 5}\right)$ & $1.68^{\mathrm{a}}, 176$ \\
$\mathrm{Si}-\mathrm{O}_{1}-\mathrm{Si}(\mathrm{deg})\left[144^{\mathrm{c}}\right]$ & $133\left(\mathbf{1 3 7}^{\mathrm{d}}\right)$ & 130 \\
$\mathrm{Si}-\mathrm{O}_{2}-\mathrm{Si}(\mathrm{deg})\left[150^{\mathrm{c}}\right]$ & $157\left(\mathbf{1 5 8}^{\mathrm{d}}\right)$ & 148 \\
$\mathrm{O}_{1}-\mathrm{Si}-\mathrm{O}_{2}(\mathrm{deg})\left[109^{\mathrm{c}}\right]$ & $105\left(\mathbf{1 0 4}^{\mathrm{d}}\right)$ & 80 \\
$\overline{\mathrm{A}}_{\text {strong }}(\mathrm{mT})$ & $\mathbf{1 4 . 7}^{\mathrm{d}}\left(11.4^{\mathrm{b}}\right)$ & \\
$\overline{\mathrm{A}}_{\text {weak }}(\mathrm{mT})$ & $\mathbf{1 . 3 0}^{\mathrm{d}}\left(1.70^{\mathrm{b}}\right)$ & \\
$\mathrm{B}_{1}(\mathrm{mT})$ & $-7.70\left(-6.47^{\mathrm{b}}\right)$ & \\
$\mathrm{B}_{2}(\mathrm{mT})$ & $3.84\left(3.23^{\mathrm{b}}\right)$ & \\
$\mathrm{B}_{3}(\mathrm{mT})$ & $3.84\left(3.23^{\mathrm{b}}\right)$ & \\
\hline
\end{tabular}

${ }^{\mathrm{a} B o n d}$ of $\mathrm{O}_{1}$ and $\mathrm{O}_{2}$ to the common $\mathrm{Si}$ neighbor.

bexperimental data taken from Reference 6.

cfrom Reference 26.

${ }^{\mathrm{d} U H F}$ results see text.

energy functionals is expressed in the Perdew-BurkeErnzerhof generalized gradient approximation (GGA). ${ }^{21}$ Wave-function optimizations are performed using the orbital transformation method. ${ }^{22}$ Explicit convergence tests suggest the usage of a double- $\zeta$ basis set and a density cutoff of 280 Ry. Moreover, in order to get an insight into the mechanism responsible for self-trapped hole hopping in amorphous silica, extensive large step quantum molecular-dynamics simulations with SIC (SIC-LSMD) are performed, using the recently developed method by Kühne et $a l .{ }^{23}$ which unifies the Car-Parrinello ${ }^{24}$ and Born-Oppenheimer MD and allows for much longer time steps (and therefore simulation times) than standard $a b$ initio without loss of accuracy.

The periodic model of amorphous silica used in the simulations is generated by quenching from the melt using a combination of classical ${ }^{25}$ and Car-Parrinello MD.

We first perform UHF calculations that are used as a reference. One electron is removed from the $\mathrm{SiO}_{2}$ model system and the structure is fully optimized. All the calculations are performed at the experimental density value keeping the volume of the supercell fixed. After geometry relaxation, the resulting hole localizes on a single bridging oxygen $\mathrm{O}_{1}$. The optimization leads to a relevant local distortion: a significant elongation of the two $\mathrm{Si}-\mathrm{O}_{1}$ bonds, that vary from the starting values of 1.61 and $1.62 \AA$ to 1.80 and $1.78 \AA$, is observed. The obtained configuration can thus be classified as STH1. The structural properties of this configuration are reported in Table I. In Ref. 11 a similar calculation has been performed on a small silica cluster without including PBC. The authors of Ref. 11 found that the Si-O bonds, after hole localization, elongated from 1.6 to $1.84 \AA$. We rationalize this difference by performing a variable cell optimization within our PBC scheme. This leads to a stable structure with the same local distortion as in Ref. 11 but the density of the system decreases from the experimental value of 2.20 to
$1.78 \mathrm{~cm} / \mathrm{gr}^{3}$, indicating that finite size and boundary effects of the previous cluster calculation ${ }^{11}$ played a major role and affected the final structure.

Although the structural properties at the experimental density and the corresponding hyperfine parameters (see below) are physically reasonable for the STH1, the fact that no attempt to represent the STH2 at UHF level of theory was successful further indicates the importance for self-trapped holes of the inclusion of correlation effects, which can be explicitly taken into account using beyond-HF theories or DFT. As already stated above, standard exchange-correlation functionals fail however in describing localization due to the nonperfect cancellation of the self-interaction term in the Coulomb energy. This can be, in part, overcome with an ad hoc self-interaction correction (SIC). Several SIC functionals have been proposed in the last decade. ${ }^{15}$ The one proposed in Ref. 16 has been employed in the study of several systems ${ }^{16,27}$ and captures the relevant physics of the considered class of systems. We adopt this functional, which adds the empirical term $E_{\mathrm{SIC}}^{\mathrm{SS}}=-a E_{H}[m]-b E_{\mathrm{XC}}[m, 0]$ to the KohnSham energy functional and contains two parameters $a$ and $b$, whereas the spin density $m=\rho_{\alpha}-\rho_{\beta}$ is exactly the density of the singly occupied orbital. The terms $E_{H}[m]$ and $E_{\mathrm{XC}}[m, 0]$, contained in $E_{\mathrm{SIC}}^{\mathrm{SS}}$ represent, respectively, the Hartree energy and an approximated exchange and correlation functional.

We explicitly verified that $b \neq 0$ would always lead to a completely delocalized hole. With $b=0$, different values of $a$ are chosen and the computed anisotropic part of the hyperfine values are compared to those in Ref. 6. Eventually, the choice of $a=0.15$ and $b=0$ (similarly in Ref. 16) appears to be the most accurate in describing self-trapped holes. With these parameters we are able to represent at a DFT level both the STH's unconstrained structures. At a SIC-DFT level a geometry optimization of the STH1 self-trapped hole leads to a significant elongation of the two Si-O bonds linked to the bridging oxygen on which the hole is localized. The final values of the interested $\mathrm{Si}-\mathrm{O}$ bonds are 1.78 and $1.80 \AA$. The structural properties of this configuration are reported in Table I.

Using the SIC-DFT approach we are also able to stabilize the STH2 defect without applying any constraints, thanks to the following procedure. The previously obtained STH1 configuration is used as a starting structure for a short (3 ps) standard Born-Oppenheimer SIC-MD simulation at room temperature. We monitor the total spin density of the system along the trajectory. The hole wave function initially sits on a single bridging $\mathrm{O}$ atom; at a certain point, spontaneously, it splits into two parts, leading to a transient configuration in which it is shared between two nearest-neighbor bridging oxygen atoms, $\mathrm{O}_{1}$ and $\mathrm{O}_{2}$, before hopping to another oxygen. This structure is extracted from the trajectory and is fully optimized with respect to the nuclear coordinates, leading to a metastable configuration with the following properties. The hole redistributes in such a way that the spin density of the two $\mathrm{O}$ atoms is $0.53 e$ and $0.40 e$, respectively. The O-Si-O angle is found to be $80^{\circ}$ and the corresponding $\mathrm{Si}-\mathrm{O}$ bond lengths are 1.67 and $1.68 \AA$, respectively. These values result to be quite close to the experimental values of $\mathrm{Si}-\mathrm{O}$ bond length in $\mathrm{SiO}_{2}$ and this configuration is identified with the 


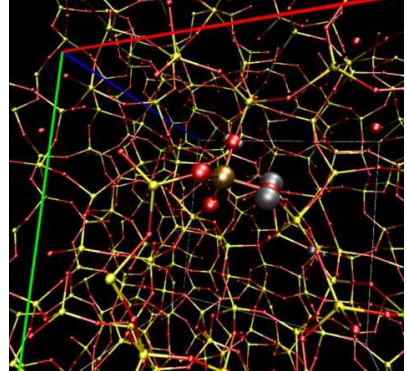

(a)

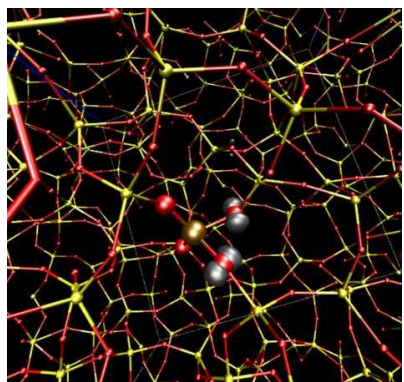

(b)

FIG. 1. (Color online) Disordered $\mathrm{SiO}_{2}$ model used in the simulations: yellow (bright) atoms are $\mathrm{Si}$ and red atoms are O. Left: STH1 configuration, with the hole localized on a single $\mathrm{O}$ with the bond to its neighboring Si strongly elongated; right: STH2 configuration with the hole shared between two oxygens. The unpaired spin density is plotted at the value of 0.2 electrons $/ \AA^{3}$.

STH2 configuration. The difference in energy between the STH1 and STH2 is found to be $-0.02 \mathrm{eV}$.

To validate our approach and to make a connection with ESR experiments, the hyperfine parameters in the STH1 structure are computed. Hyperfine interaction includes an isotropic part (Fermi contact) and an anisotropic (dipolar) part. ${ }^{15}$ The isotropic part measures the spin density at the nucleus and it is nonzero only for wave functions containing $s$-wave components. The hole state, both in the localized or delocalized states, corresponds to the $\mathrm{O} 2 p$ lone-pair state. Thus the Fermi contact is mainly due to the spin polarization of the doubly occupied O $2 s$ state. Since the spin-restricted conditions suppress such spin polarization in the SIC formulation, the Fermi-contact term cannot be computed within DFT-SIC, ${ }^{15}$ but only in the UHF calculations. We compute the mean eigenvalues of the strong and weak components $\overline{\mathrm{A}}_{\text {strong }}$ and $\overline{\mathrm{A}}_{\text {weak }}$ of the hyperfine tensor. The hyperfine tensor is a tensor of rank 2, which describes the coupling between the electronic spin and the nuclear spin of a given nucleus. The strongest feature $\overline{\mathrm{A}}_{\text {strong }}$ corresponds to the $\mathrm{O}$ atom on which the electron is localized while the $\overline{\mathrm{A}}_{\text {weak }}$ corresponds to the nearest-neighbor $\mathrm{O}$ atoms. The computed mean eigenvalues are reported in Table I and found in fair agreement with the experimental data in Ref. 6. In the DFTSIC approach, instead, we could access only the anisotropic terms since they capture the $p$-like component of the electron wave function which is weakly affected by the spin-restricted condition. The evaluated anisotropic part of the hyperfine values $\left(B_{1}, B_{2}\right.$, and $\left.B_{3}\right)$ in the STH1 case are found in agreement with the experiment, as shown in Table I.

Having established the nature of the STH1 and STH2 ground states, the last part of this work is devoted to the study of the hopping mechanism. The characterization of this microscopic event paves the way to the understanding of the macroscopic degradation of silica-based semiconductor devices. To this end, extensive SIC-LSMD with a time step of $2 \mathrm{fs}$ are performed using as starting configuration the STH1 and STH2 structures. The resulting lengths of the simulations are 160 and $50 \mathrm{ps}$, respectively. The spin population of the $\mathrm{O}$ atoms is monitored along the simulations. A typical scenario occurring during the simulation is the migration of the un-

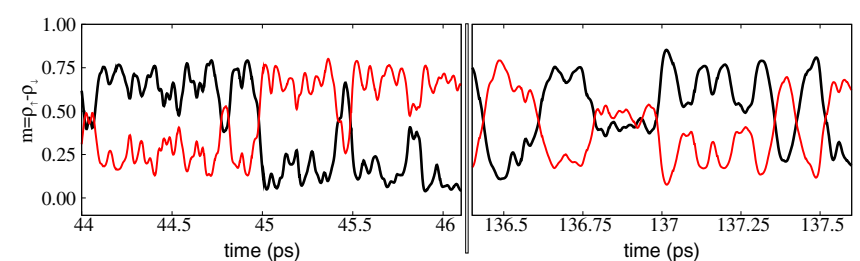

FIG. 2. (Color online) Sections of the quantum LSMD trajectory showing typical hopping mechanisms observed in the simulations. The two lines correspond to the spin population of the atoms involved in the hopping mechanism: $\mathrm{O}_{1}$ (black) and its nearestneighbor $\mathrm{O}_{2}$ (red). The hole initially localized on $\mathrm{O}_{1}$ STH1 suddenly hops to $\mathrm{O}_{2}$ (dark gray line) $(t=44.8)$. The spin population of $\mathrm{O}_{1}$ decreases from an average value of $0.75 e$ to an average value of $0.07 e$ (left panel). At $t=136.76$ the hole initially localized on $\mathrm{O}_{2}$ hops to $\mathrm{O}_{1}$, and is shared by $\mathrm{O}_{2}$ and $\mathrm{O}_{1}$ for 0.3 ps (STH2), then it jumps again to the $\mathrm{O}_{2}$ atom (right panel).

paired electron (hole) from the STH1 to the STH2 and to the STH1 configuration again. The hole localized on a single nonbridging oxygen $\mathrm{O}_{1}$ in the STH1 configuration splits into two parts, one remaining on the $\mathrm{O}_{1}$ oxygen and one migrating to the nearest-neighbor oxygen atom $\mathrm{O}_{2}$, resulting in a STH2; then it completely migrates to $\mathrm{O}_{2}$ atom, see Figs. 1 and 2. The hopping mechanism happens with a significant change in the value of the $\mathrm{O}_{1}-\mathrm{Si}-\mathrm{O}_{2}$ angle, which varies from the value of 100 to $80^{\circ}$ in agreement with the results of Pacchioni et al. ${ }^{11}$ The time that the hole spends sitting on a single bridging $\mathrm{O}_{2}$ oxygen atom can vary from fractions of picosecond to $\sim 1$ picosecond, see Fig. 2. In total we observe $\sim 300$ hopping events along the simulation, which correspond to a maximum time of 2 ps spent by the hole on a single $\mathrm{O}$ atom. This picture is in agreement with the explanation proposed by Griscom: the hole is not delocalized between two $\mathrm{O}$ atoms but hops between them very rapidly, and the delocalized hole can be seen as an average structure between two rapidly interchanging states where the hole hops between $\mathrm{O}_{1}$ and $\mathrm{O}_{2}$.

Another extremely interesting phenomenon has been observed during the simulation. In Fig. 3 we show a plot of the time evolution of the spin population of three couples of nearest-neighbor $\mathrm{O}$ atoms. The hole, initially shared between the $\mathrm{O}_{1}$ and $\mathrm{O}_{2}$ atoms, moves ( $t=36$ ps) to another couple of oxygen atoms $\mathrm{O}_{3}$ and $\mathrm{O}_{4}$ at a distance of $\sim 4 \AA$ and then again $(t=36.8 \mathrm{ps})$ to a third couple $\mathrm{O}_{5}$ and $\mathrm{O}_{6}$ at a distance of $3.7 \AA$, where it is shared for 25 ps (not shown).

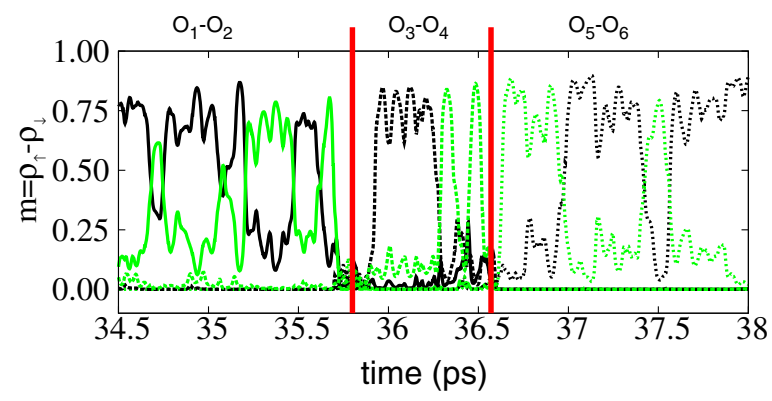

FIG. 3. (Color online) Section of the quantum LSMD trajectory showing the mechanism for the hole hopping between three couples of bridging $\mathrm{O}$ atoms at a $3.97 \AA$ distance. 
It is important to note that the hole hopping or tunneling are not properly described within the Born-Oppenheimer approximation. Since the time scales for the electron dynamics, in this case, are comparable with the nuclear ones, no classical separation of the ionic movement can be assumed. A more detailed description of the mechanism would require the diabatic treatment of both atomic and electronic degrees of freedom at a quantum-mechanical level. For the similar case of electron transfer, several methods have been developed (for example, in Ref. 28 a method based on penalty functionals was successfully applied to ferrous and ferric ions in water) and a diabatic description would be appropriate in this case as well. Nevertheless, we consider our results as promising. Indeed, the present treatment based on selfinteraction corrected DFT already gives reliable results, which are compatible with the experiments. Moreover, the structural quantities extracted about the two kinds of observed defects are physically sound and complete and extend previous studies on the same subject.
In summary, we have used unrestricted HF and SIC-DFT calculations to reproduce self-trapped holes in a disordered $\mathrm{SiO}_{2}$ network and we have performed molecular-dynamics simulations to investigate the mechanism by which the hole hops between nearest-neighbor bridging oxygen atoms. The experimental predicted structure ${ }^{6}$ of the STH2 has been, for the first time, reproduced at a density functional theory level. These results, together with an insight into the hole hopping mechanism, should contribute to a deeper and better understanding of damage mechanisms involving amorphous materials.

We are grateful to Erio Tosatti for fruitful discussions and careful reading of the manuscript and we thank Alfredo Pasquarello and Carlo Pignedoli for constructive suggestions. We thank U. Sennhauser and L. Schlapbach who provided the motivation for the present work. We acknowledge computational resources from the Swiss Supercomputing Centre in Manno.
${ }^{1}$ D. L. Griscom, Phys. Rev. B 40, 4224 (1989).

${ }^{2}$ W. Hayes and T. J. L. Jenkin, J. Phys. C 19, 6211 (1986).

${ }^{3}$ Physics of Color Centers, edited by W. B. Fowler (Academic, New York, 1968).

${ }^{4}$ T. G. Castner and W. Känzig, J. Phys. Chem. Solids 3, 178 (1957).

${ }^{5}$ N. F. Mott, Adv. Phys. 26, 363 (1977).

${ }^{6}$ D. L. Griscom, J. Non-Cryst. Solids 149, 137 (1992).

${ }^{7}$ P. V. Chernov, E. M. Dianov, V. N. Karpechev, L. S. Kornienko, I. O. Morozova, A. O. Rybaltovskii, V. O. Sokolov, and V. B. Sulimov, Phys. Status Solidi B 155, 663 (1989).

${ }^{8}$ A. H. Edwards, Phys. Rev. Lett. 71, 3190 (1993).

${ }^{9}$ A. V. Kimmel, J. Non-Cryst. Solids 353, 599 (2007).

${ }^{10}$ G. Pacchioni and A. Basile, Phys. Rev. B 60, 9990 (1999).

${ }^{11}$ S. Sicolo, G. Palma, C. Di Valentin, and G. Pacchioni, Phys. Rev. B 76, 075121 (2007).

${ }^{12}$ C. Lee, W. Yang, and R. G. Parr, Phys. Rev. B 37, 785 (1988).

${ }^{13}$ A. D. Becke, J. Chem. Phys. 98, 5648 (1993).

${ }^{14}$ Modified 6-21G(d) (Si atoms) and 6-31G(d) (O atoms) basis sets were used, http://www.crystal.unito.it/

${ }^{15}$ M. d'Avezac, M. Calandra, and F. Mauri, Phys. Rev. B 71, 205210 (2005).

${ }^{16}$ J. VandeVondele and M. Sprik, Phys. Chem. Chem. Phys. 7, 1363 (2005).

${ }^{17}$ G. Lippert, J. Hutter, and M. Parrinello, Mol. Phys. 92, 477 (1997).

${ }^{18} \mathrm{http}: / / \mathrm{cp} 2 \mathrm{k} \cdot$ berlios.de/
${ }^{19}$ S. Goedecker, M. Teter, and J. Hutter, Phys. Rev. B 54, 1703 (1996).

${ }^{20}$ M. Krack, Theor. Chem. Acc. 114, 145 (2005).

${ }^{21}$ J. P. Perdew, K. Burke, and M. Ernzerhof, Phys. Rev. Lett. 77, 3865 (1996).

${ }^{22}$ J. VandeVondele and J. Hutter, J. Chem. Phys. 118, 4365 (2003).

${ }^{23}$ T. D. Kühne, M. Krack, F. R. Mohamed, and M. Parrinello, Phys. Rev. Lett. 98, 066401 (2007).

${ }^{24}$ R. Car and M. Parrinello, Phys. Rev. Lett. 55, 2471 (1985).

${ }^{25}$ B. W. H. van Beest, G. J. Kramer, and R. A. van Santen, Phys. Rev. Lett. 64, 1955 (1990).

${ }^{26}$ The model system contains 72 atoms at the measured density of $2.20 \mathrm{~g} / \mathrm{cm}^{3}$. The side of the periodically repeated cubic supercell is $10.29 \AA$. In Ref. 26 we compared to other model systems (Ref. 29) and experimental data the structural and electronic properties of this model that was also employed to study point defects (Ref. 30). The electronic structure has been treated using DFT theory at a GGA level, submitted to J. Non-Cryst. Solids.

${ }^{27}$ Y. A. Mantz, F. L. Gervasio, T. Laino, and M. Parrinello, Phys. Rev. Lett. 99, 058104 (2007).

${ }^{28}$ P. H.-L. Sit, M. Cococcioni, and N. Marzari, Phys. Rev. Lett. 97, 028303 (2006).

${ }^{29}$ J. Sarnthein, A. Pasquarello, and R. Car, Phys. Rev. B 52, 12690 (1995).

${ }^{30}$ M. Farnesi Camellone, J. C. Reiner, U. Sennhauser, and L. Schlapbach, Phys. Rev. B 76, 125205 (2007). 